\title{
Surface spin-flop phases and bulk discommensurations in antiferromagnets
}

\author{
C Micheletti†, R B Griffiths $\ddagger$ and J M Yeomans $†$ \\ $\dagger$ Theoretical Physics, Oxford University, 1 Keble Road, Oxford OX1 3NP, UK \\ $\ddagger$ Physics Department, Carnegie-Mellon University, Pittsburgh, PA 15213
}

(November 1996)

\begin{abstract}
Phase diagrams as a function of anisotropy $D$ and magnetic field $H$ are obtained for discommensurations and surface states for a model antiferromagnet in which $H$ is parallel to the easy axis. The surface spin-flop phase exists for all $D$. We show that there is a region where the penetration length of the surface spin-flop phase diverges. Introducing a discommensuration of even length then becomes preferable to reconstructing the surface. The results are used to clarify and correct previous studies in which discommensurations have been confused with genuine surface spin-flop states.
\end{abstract}

Recent experimental results on $\mathrm{Fe} / \mathrm{Cr}(211)$ superlattices [1,2] have stimulated theoretical and numerical work aimed at explaining the occurrence of series of phase transitions in samples with an even number of Fe blocks. The antiferromagnetically-coupled Fe blocks can be described phenomenologically by a chain of classical X-Y spins with antiferromagnetic interactions and uniaxial spin anisotropy $D$. For a chain of infinite length, application of an external magnetic field $H$ in a direction parallel to the easy axis (the spin direction in zero field) results in a transition at a finite field $H=H_{S F}(D)$ to a spin-flop phase in which the spins on the two sublattices are approximately perpendicular to the applied field, and nearly opposite to each other [3].

For finite chains the situation is more complex. In 1968 Mills [4] suggested that spins in a layer near a free surface of such an antiferromagnet could rotate into the flopped state at a field $H_{S F}^{\prime}$ significantly less than $H_{S F}$. To understand this further we present numerical and analytic studies for an antiferromagnetic chain for all values of $H$ and $D$. We show that a consideration of both surface states and discommensurations is necessary to fully understand the phase diagram. It transpires that discommensurations, while they are not surface phenomena per se can provide the minimum energy configuration for both semi-infinite and finite systems. The results allow us to unify certain aspects of previous work [1, 2, 1, 7] while correcting others.

Consider a chain of classical spins described by a Hamiltonian

$$
\mathcal{H}=\sum_{i}\left[\cos \left(\theta_{i}-\theta_{i+1}\right)-H \cos \theta_{i}-\frac{D}{4} \cos \left(2 \theta_{i}\right)\right],
$$

where the exchange coefficient has been taken as the unit of energy, $\theta_{i}$ is the angle between the $i$ th spin and the direction of the field $H$, and $D$ is a two-fold spin anisotropy. One can think of $\theta_{i}$ as the direction of the magnetization in the $i$ th layer of an antiferromagnet, or in the $i$ th Fe layer in a Fe/Cr superlattice. It is appropriate to work at zero temperature in one dimension because in-plane fluctuations are not important for the superlattice physics.

For a bulk system, in which the sum in (1) runs from $-\infty$ to $+\infty$, the ground state is either ferromagnetic (F) with all spins parallel to the field, antiferromagnetic (AF) with the spins alternating between 0 and $\pi$, parallel and antiparallel to the field, or a spin flop (SF) phase in which the spins alternate between $+\phi$ and $-\phi$, for some $\phi$ in the range $0<\phi<\pi / 2$. The regions where these different phases are stable are indicated in Fig. 1, where the AF phase is above the line $D(4-D)=H^{2}$ and to the left of $H=2$, while the $\mathrm{F}$ and SF phases are separated by the line $D+H=4$ [8]. The additional structure in the AF region refers to discommensurations and surface phases.

We first describe the phase diagram of a chain which is constrained by suitable boundary conditions to include a discommensuration [9]. Note that the bulk AF ground state is degenerate with two possibilities: $\theta_{i}$ equal to 0 for $i$ even and $\pi$ for $i$ odd, or vice versa. A discommensuration is a boundary or interface between two regions corresponding to these two possibilities; in particular, a configuration in which $\theta_{2 n}$ tends to 0 and $\theta_{2 n+1}$ to $\pi$ as $n$ tends to $+\infty$, while $\theta_{2 n}$ tends to $\pi$ and $\theta_{2 n+1}$ to 0 as $n$ tends to $-\infty$. For all values of $D$ above the dashed line in Fig. 1, the discommensuration of minimum energy is of the Ising type, $\ldots 0, \pi, 0, \pi, 0,0, \pi, 0, \pi, 0 \ldots$, with two adjacent spins parallel to each other and to the field, in the middle of what is otherwise an antiferromagnetic configuration.

In the AF region below the dashed line in Fig. 1, "flopped" discommensurations of different length have lower energies than the Ising discommensuration. A flopped discommensuration of type $\langle 2 m\rangle$ consists of a "core" of $2 m$ spins in which the spin configuration resembles that in a bulk SF phase, located between "tails", each of which rapidly reverts to the configuration of the corresponding AF phase with increasing distance from the core. See, for example, Fig. 1 in 10]. As $D$ decreases, the distinction between the "tails" and the "core" is less clear, but we continue to use the same label for the discommensuration which evolves continuously from $\langle 2 m\rangle$ at larger $D$. One can think of 
such a discommensuration as composed of a pair of $\mathrm{AF}-\mathrm{SF}$ and $\mathrm{SF}-\mathrm{AF}$ interfaces centered at the points where the corresponding tail joins the core. At low values of $H$, the discommensuration $\langle 2\rangle$ has the lowest energy, but upon approaching the bulk AF:SF phase boundary, one finds - see Fig. 2 for details omitted from Fig. 1 - a sequence of phase transitions to $\langle 4\rangle,\langle 6\rangle, \ldots$ as $H$ increases. Our numerical procedures, which used effective potential techniques [11 13 to construct surface states by extrapolating from the bulk [14], found values of $2 m$ up to 14 . We were able to trace the first-order lines separating the different $\langle 2 m\rangle$ phases down to a value of $D$ between 0.1 and 0.4 . We found no evidence to suggest that these lines end in critical points, and we believe it likely that they will persist all the way down to $D=0$.

These transitions reflect the discrete nature of the spin chain. Thus it is not surprising that they are absent in the continuum approximation employed in 10] for small values of $D$. That study showed that the width of the discommensuration tends to $\infty$ at the AF:SF boundary, in agreement with what we found for larger values of $D$.

The boundary between $\langle 2\rangle$ and the Ising discommensuration is second order, and represents the limit of stability of the latter as $D$ decreases. An analytic calculation yields the equation $(D+H-1)^{-1}=5 / 3+D-H$, in good agreement with our numerical calculations, and those in [10] when $H=0$. The boundaries between the Ising discommensuration and $\langle 4\rangle,\langle 6\rangle$ etc., are first order, and were obtained numerically. The triple points at which the phases $\mathrm{AF}_{2},\langle 2 m\rangle$ and $\langle 2 m+2\rangle$ meet tend to an accumulation point, $Q$, located at $H \approx 1.58, D \approx 0.78$. Presumably this is where the energy to create a pair of $\mathrm{AF}-\mathrm{SF}$ and $\mathrm{SF}-\mathrm{AF}$ interfaces (infinitely far apart) is equal to the energy of an Ising discommensuration.

We now consider the surface states of a semi-infinite chain. If a ground-state configuration of an infinite chain is cut in two while the spins are held fixed, there results two ideal or unreconstructed surfaces. Sometimes the energy of the surface state can decrease through a local reconstruction in which spins near the surface are altered from their bulk values by amounts which tend asymptotically to zero with increasing distance from the surface. The energy change during such a reconstruction can be calculated from the appropriate Hamiltonian, which is (11) with the sum going from $i=0$ to $+\infty$, even though the total energy is not well defined. Hence the reconstructed surface of minimum energy is (usually) well-defined.

While the surface reconstruction in the spin-flop phase occurs smoothly, so that there are no phase transitions, the situation in the case of antiferromagnetic surface states is more complicated. There are two types of surface states, $A$ and $B$, with unreconstructed versions having the surface spin parallel $\left(\theta_{0}=0\right)$ or opposite $\left(\theta_{0}=\pi\right)$ to the field direction respectively. Even after reconstruction takes place, so that $\theta_{0}$ has changed, the $A$-type ( $B$-type) surface can still be identified through the fact that $\theta_{2 n}$ tends to $0(\pi)$ as $n \rightarrow \infty$.

Throughout the AF region, $A$-type surfaces do not reconstruct. The behavior of $B$-type surfaces is more complicated. In region $\mathrm{AF}_{1}$ in Fig. 1, the unreconstructed surface has the lowest energy. In region $\mathrm{AF}_{2}$, which meets $\mathrm{AF}_{1}$ along a line $H=1$ for arbitrarily large $D$, there is a set of degenerate (equal minimum energy) reconstructed surfaces,

$$
[0\rangle=00 \pi 0 \pi 0 \pi \ldots, \quad[2\rangle=0 \pi 00 \pi 0 \pi \ldots,
$$

where [2n> consists of $2 n$ spins in an antiferromagnetic arrangement, followed by two spins parallel to the field, and then the bulk antiferromagnetic phase. One can think of this reconstructed surface as an Ising discommensuration located a certain distance from the surface. Because the "tails" of this discommensuration have zero length, it does not interact with the surface, and its energy is independent of its distance from the surface as long as $\theta_{0}=0$. (This artificial degeneracy would be lifted if weak longer-range interactions were included in (1).)

In the $\mathrm{AF}_{3}$ region in Fig. 1, the $B$-type surface again reconstructs, but the spins near the surface are no longer locked in Ising positions. Just as in the $\mathrm{AF}_{2}$ region, one can think of the surface state as consisting of a discommensuration located a finite distance from the surface, but now this discommensuration is of the flopped type with a core of length two, and tails extending out on either side of the core. Because of the tails, the discommensuration interacts with the surface, and the minimum surface energy occurs when it is some specific distance $2 n$ from the surface, depending on $D$ and $H$. Thus in $\mathrm{AF}_{3}$ one finds genuine spin-flop surface states. As $H$ increases, the discommensuration moves further from the surface. It does this, at least when $D$ is large, discontinuously in steps of 2, via a series of first-order phase transitions, some of which are shown in Fig. 2, where they extend leftwards from the point $P$. Numerically we have seen states with $2 n$ up to 14 , and our results are consistent with $n$ tending to infinity at the right side of the $\mathrm{AF}_{3}$ region, which our analytic calculations, in agreement with [5], show to be the line

$$
D=\sqrt{1+H^{2}}-1 \text {. }
$$

The upper boundary of the $\mathrm{AF}_{3}$ region extending from $O$ to $P$ is a continuous (second-order) transition representing the limit of stability of the Ising surface phase [0 , see (2]), as $D$ decreases. An analytic calculation yields the implicit equation

$$
(2+D-H-1 / a)^{-1}=2+D+H-a,
$$




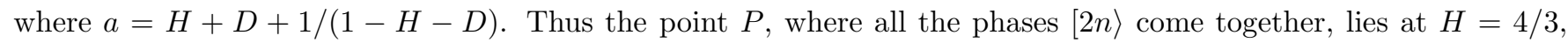
$D=2 / 3$, the intersection of (3) and (4). Both (3) and (4) agree with our numerical results.

We find that the first-order lines extending downwards and leftwards from $P$ in Fig. 2, separating the canted phases $[2 n\rangle$ from $[2 n+2\rangle$, end in critical points as $D$ decreases. The larger the value of $n$, the further the line extends towards the origin, but presumably for any finite value of $n$ the difference between the phases $[2 n\rangle$ and $[2 n+2\rangle$ eventually disappears at some finite value of $D$. Because this value decreases with increasing $n$, it is plausible that the corresponding critical points accumulate at the origin.

As is evident in Fig. 1, the region $\mathrm{AF}_{3}$ becomes extremely narrow as $D$ decreases. The left boundary approaches a parabola $D=0.5 \mathrm{H}^{2}$ to within numerical precision, which is asymptotically the same as (3). We nonetheless believe that the width of $\mathrm{AF}_{3}$ remains finite as long as $D>0$. Numerical evidence for this is that the value of the surface spin $\theta_{0}$, at the left edge of the $A F_{3}$ region (that is for $H$ just large enough to produce the surface spin-flop phase) tends to a value near $\pi / 3$ as $D$ goes to zero. This indicates that even for very small $D$ the discommensuration at the threshold field is still a finite distance from the surface.

Between $\mathrm{AF}_{3}$ and the $\mathrm{AF}: \mathrm{SF}$ bulk phase boundary lies region $\mathrm{AF}_{4}$ in which the flopped discommensuration is repelled by the surface, so that its minimum energy location is in the bulk infinitely far away from the surface, as noted in ref. [5]. Thus there is not a minimum-energy reconstructed $B$ surface, or, properly speaking, a "surface spin-flop phase" in region $\mathrm{AF}_{4}$. It would probably be better to identify $\mathrm{AF}_{4}$, thought of as part of the $B$-type surface phase diagram, as the "discommensuration phase", since the minimum energy surface will always be of the $A$-type.

In retrospect it seems likely that the broadening of the SSF transition mentioned in the abstract of ref. [6] actually refers to broadening of the bulk discommensuration which, as noted above, occurs as $H$ approaches the AF:SF boundary inside region $\mathrm{AF}_{4}$. It seems that no earlier work has correctly identified the stable SSF phase at small values of $D$, characterized when it first appears with increasing $H$ by a surface spin with a value very near $\pi / 3$. The narrowness of the $\mathrm{AF}_{3}$ region for small $D$ may be why it was overlooked.

For a finite chain of length $L$, the minimum energy is achieved with $A$-type surfaces at both ends when $L$ is odd. This is not possible if $L$ is even, so when $H$ is small there will be an $A$-type surface at one end and a $B$-type at the other. As $H$ increases, the latter reconstructs through a discommensuration which then moves towards the centre, where it arrives when the value of $H$ is approximately that given by (3). Next the discommensuration proceeds to broaden in discontinuous steps, with the AF-SF and SF-AF interfaces on the left and right side of the core moving outwards, until they arrive at the two surfaces when $H$ is approximately at the bulk AF:SF transition value. The numerical results reported in [1.2, (7) for even values of $L \geq 16$ and $D=0.5$ agree well with the phase diagrams in Figs. 1 and 2 . The same broadening scenario for the discommensuration, at a much smaller value of $D$, is visible in Fig 3 of [5].

JMY and CM acknowledge support from the EPSRC. We thank D. L. Mills for bringing [10] to our attention.

[1] R. W. Wang, D. L. Mills, E. E. Fullerton, J. E. Mattson and S. D. Bader, Phys. Rev. Lett. 72, 920 (1994).

[2] R. W. Wang and D. L. Mills, Phys. Rev. B 503931 (1994).

[3] L. Néel, Ann. Phys. (Paris) 5, 232 (1936)

[4] D. L. Mills, Phys. Rev. Lett. 20, 18 (1968).

[5] L. Trallori, P. Politi, A. Rettori, M. G. Pini and J. Villain, Phys. Rev. Lett. 72, 1925 (1994).

[6] F. Keffer and H. Chow, Phys. Rev. Lett. 31, 1061 (1973).

[7] L. Trallori, P. Politi, A. Rettori, M. G. Pini and J. Villain, J. Phys. C 7, L451 (1995).

[8] F. B. Anderson and H. B. Callen, Phys. Rev. A, 136, 1068 (1964)

[9] For detailed studies of discommensurations in Frenkel-Kontorova models see, for example, S. Aubry, in Solitons and Condensed Matter Physics, edited by A. R. Bishop and T. Schneider (Springer Verlag, Berlin, 1981), p. 264; J. Phys. (Paris) 44, 147 (1983); Physica D 7, 240 (1983); also R. B. Griffiths, in Fundamental Problems in Statistical Mechanics VII, edited by H. van Beijeren (Elsevier, Amsterdam, 1990), p. 69.

[10] N. Papanicolaou, Phys. Rev. B 51, 15062 (1995).

[11] W. Chou and R. B. Griffiths, Phys. Rev. B 34, 6219 (1986).

[12] L. M. Floria and R. B. Griffiths, Numer. Math. 55, 565 (1989).

[13] K. Hood, J. Comput. Phys. 89, 187 (1990).

[14] C. Micheletti, D. Phil. thesis, Oxford University (1996) 


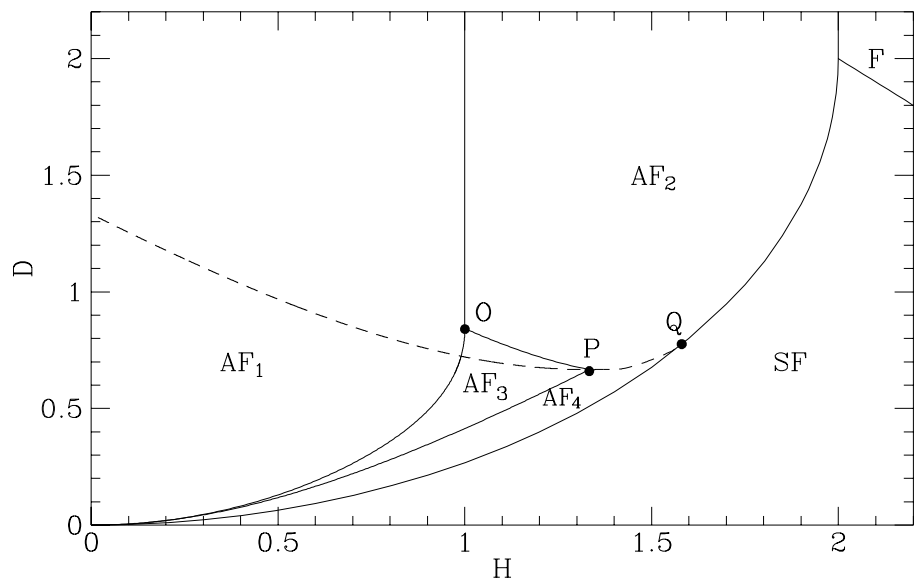

FIG. 1. Phase diagram in the $H, D$ plane. Additional details are in Fig. 2.

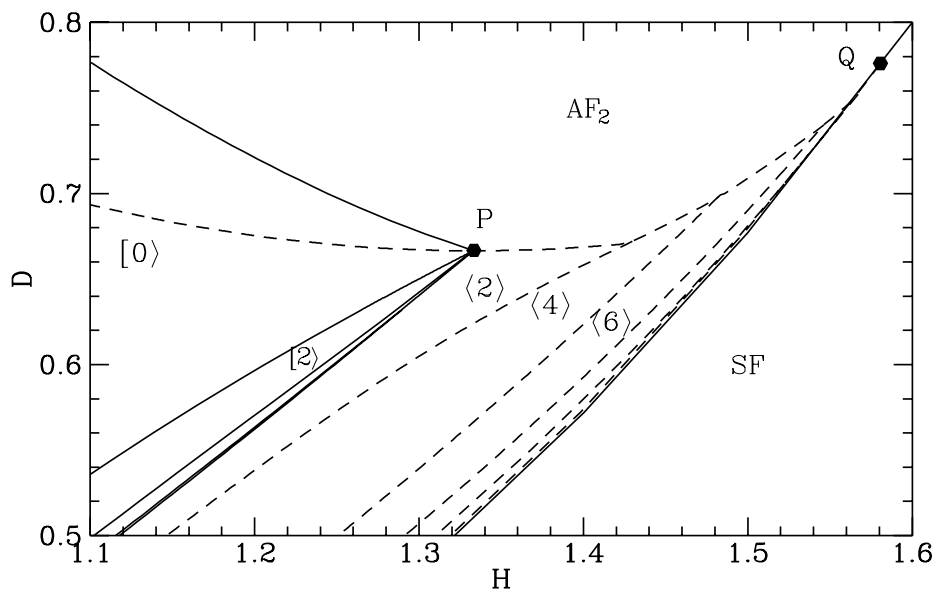

FIG. 2. Phase diagram: details near the points $P$ and $Q$. 